\title{
Blasius Boundary Layer Solution With Slip Flow Conditions
}

\author{
Michael J. Martin and Iain D. Boyd \\ Department of Aerospace Engineering \\ University of Michigan \\ Ann Arbor, MI 48109-2140
}

\begin{abstract}
As the number of applications of micro electro mechanical systems, or MEMS, increase, the variety of flow geometries that must be analyzed at the micro-scale is also increasing. To date, most of the work on MEMS scale fluid mechanics has focused on internal flow geometries, such as microchannels. As applications such as micro-scale flyers are considered, it is becoming necessary to consider external flow geometries. Adding a slip-flow condition to the Blasius boundary layer allows these flows to be studied without extensive computation.
\end{abstract}

\section{BOUNDARY LAYER WITH SLIP}

The Blasius boundary layer solution for flow over a flat plate is among the best know solutions in fluid mechanics [1]. The boundary layer equations assume the following: (1) steady, incompressible flow, (2) laminar flow, (3) no significant gradients of pressure in the x-direction, and (4) velocity gradients in the $\mathrm{x}$ direction are small compared to velocity gradients in the y-direction. Only the last assumption is questionable for MEMS scale flows.

\section{No-Slip Boundary Layer Equations}

The simplified Navier-Stokes Equations based on these assumptions, known as the boundary layer equations, are given as:

$$
\begin{gathered}
\frac{\partial u}{\partial x}+\frac{\partial v}{\partial y}=0 \\
u \frac{\partial u}{\partial x}+v \frac{\partial u}{\partial y}=v \frac{\partial^{2} u}{\partial y^{2}}
\end{gathered}
$$

where $u$ and $v$ are the $x$ and $y$ components of the velocity, and $v$ is the kinematic viscosity of the fluid.

In the Blasius solution, a non-dimensional position $\eta$ combines both the $\mathrm{x}$ and $\mathrm{y}$ position:

$$
\eta=\frac{y^{*}}{\left(x^{*}\right)^{1 / 2}}=\frac{y /\left(\mathrm{v} \mathrm{L} / \mathrm{u}_{0}\right)^{1 / 2}}{(\mathrm{x} / \mathrm{L})^{1 / 2}}=\frac{y}{\left(\mathrm{v} \mathrm{x} / \mathrm{u}_{0}\right)^{1 / 2}}
$$

where $\mathrm{x}^{*}$ and $\mathrm{y}^{*}$ are non-dimensional coordinates, $\mathrm{u}_{\mathrm{o}}$ is the free stream velocity, and $\mathrm{L}$ is an arbitrary length scale that cancels itself out. The non-dimensional velocities $u^{*}$ and $v^{*}$ are then functions of the nondimensional stream function $f$ :

CP585, Rarefied Gas Dynamics: 22 $2^{\text {nd }}$ International Symposium, edited by T. J. Bartel and M. A. Gallis (C) 2001 American Institute of Physics 0-7354-0025-3/01/\$18.00 


$$
u^{*}=u / u_{o}=f^{\prime}(\eta), v^{*}=v /\left(v u_{o} / x\right)=0.5\left[\eta f^{\prime}(\eta)-f(\eta)\right]
$$

A governing equation for $f$ can be found by substituting (3) and (4) into the $\mathrm{x}$-momentum equation (2):

$$
f^{\prime \prime \prime}(\eta)-0.5 f(\eta) f^{\prime}(\eta)=0
$$

For flow at non-rarefied length scales, the boundary conditions for the problem are no-slip, and no through flow at the wall, and $u=u_{0}$ as $y$ approaches infinity. In non-dimensional variables, these become:

$$
\begin{aligned}
& u *(y=0)=0 \Rightarrow f^{\prime}(\eta=0)=0 \\
& v *(y=0)=0 \Rightarrow f(\eta=0)=0 \\
& u *(y \rightarrow \infty)=1 \Rightarrow f^{\prime}(\eta \rightarrow \infty)=1
\end{aligned}
$$

\section{Slip Boundary Conditions}

When the flow becomes rarefied, the no-slip condition (6) at the wall is replaced by a slip-flow condition [2]. For an isothermal wall, the slip condition is given by

$$
\mathbf{u}_{\text {wall }}=\left.\frac{(2-\sigma)}{\sigma} \lambda \frac{\partial \mathrm{u}}{\partial \mathrm{y}}\right|_{\text {wall }}
$$

where $\lambda$ is the mean free path, and $\sigma$ is the tangential momentum accommodation coefficient. This can be non-dimensionalized to obtain

$$
f^{\prime}(0)=\frac{(2-\sigma)}{\sigma} \operatorname{Kn}_{x} \operatorname{Re} x^{1 / 2} f^{\prime \prime}(0)=\mathrm{K}_{1} f^{\prime \prime}(0)
$$

where $\mathrm{Kn}_{\mathrm{x}}$ and $\mathrm{Re}_{\mathrm{x}}$ are the Knudsen and Reynolds numbers based on $\mathrm{x}$, and $\mathrm{K}_{1}$ is a non-dimensional parameter that describes the behavior at the surface:

$$
\mathrm{K}_{1}=\frac{(2-\sigma)}{\sigma} \mathrm{Kn}_{\mathrm{x}} \operatorname{Re} x^{1 / 2}
$$

\section{NUMERICAL SOLUTION}

These equations are solved using a shooting method, just as the no-slip boundary layer equations are solved. There is one unique value of $f^{\prime \prime}(0)$ and $f^{\prime}(0)$ for each value of $\mathrm{K}_{1} \cdot f^{\prime \prime}(0)$ is shown in figure 1:

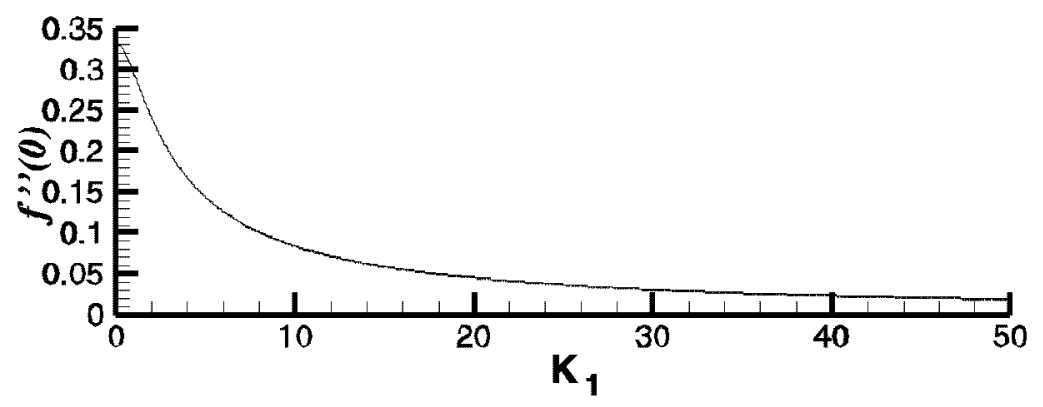

Figure 1.f" $(0)$ versus $\mathrm{K}_{1}$ 
As the analysis is expanded to large values of $\mathrm{K}_{1}, f^{\prime \prime}(0)$ will asymptotically approach zero. Figure 2 shows $f^{\prime}(0)$, or the non-dimensional slip velocity, as a function of $\mathrm{K}_{1}$ :

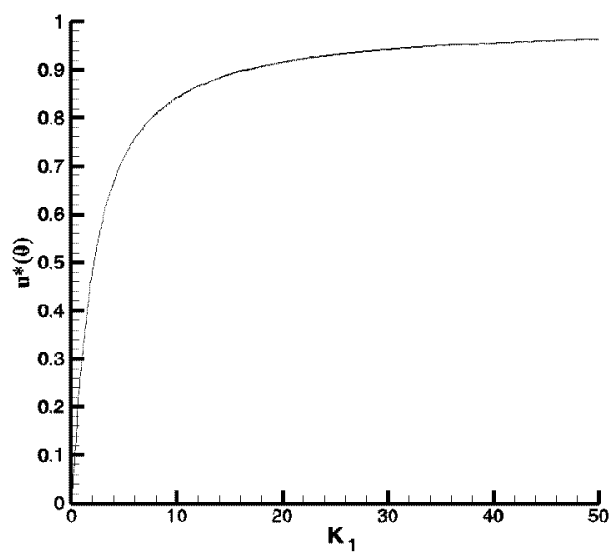

Figure 2. $u^{*}{ }_{\text {wall }}$ versus $\mathrm{K}_{1}$

As the Knudsen number approaches zero, $\mathrm{K}_{1}$ also approaches zero, and the no-slip condition, and the classical boundary layer solution, are recovered. As the Knudsen number becomes large, $\mathrm{K}_{1}$ approaches infinity, and the non-dimensional slip velocity approaches 1 , indicating 100 percent slip at the wall.

The velocity profile within the boundary layer will also change as a function of $K_{1}$. Because the initial value of $f$ " changes as we move along the plate, the self-similarity of the Blasius solution is lost. However, because conservation of mass and momentum are satisfied in the same approximate manner as in the Blasius solution, the approach remains valid. Figure 3 below shows the normalized velocity profile in the boundary layer for various values of $\mathrm{K}_{1}$ :

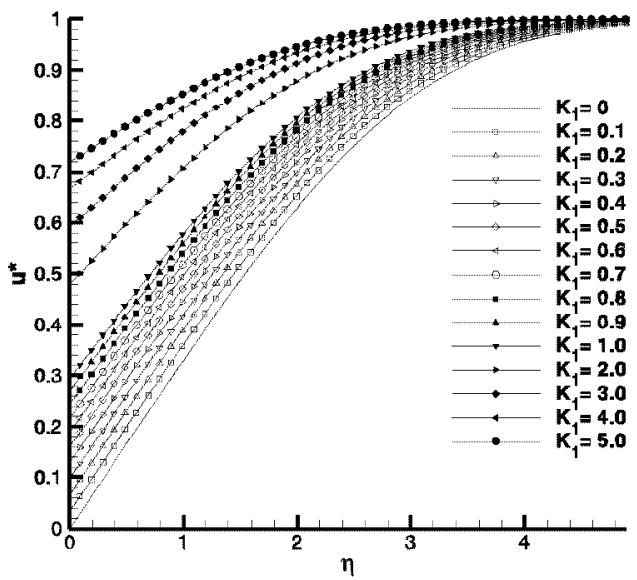

Figure 3. $u^{*}$ versus $\eta$ for various values of $K_{1}$

One result that can be seen in figure 3 is that even as the wall velocity changes drastically, the overall boundary layer thickness does not change as rapidly. The physical thickness of the boundary layer is

$$
\frac{\delta_{99}}{x}=\eta_{99} \operatorname{Re}_{x}^{1 / 2}
$$


Equation (12) can be substituted into (11) to obtain a $\mathrm{K}_{1}$ based on boundary layer thickness:

$$
K_{1}=\frac{(2-\sigma)}{\sigma} \frac{K n_{\delta}}{\eta_{99}}
$$

For equilibrium flows, $\eta_{99}$ is a constant with a value of 4.9 . For a non-equilibrium boundary layer, $\eta_{99}$ varies along the plate. Figure 4 shows the value of $\eta_{99}$, where $u^{*}$ is equal to .99 , as a function of $K_{1}$ :

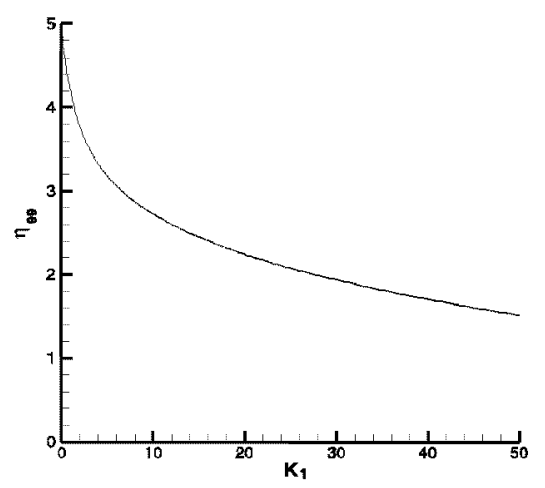

Figure 4. $\eta_{99}$ versus $\mathrm{K}_{1}$

The non-equilibrium behavior at the wall, as measured by $\mathrm{K}_{1}$, will be proportional to the boundary layer thickness, which is a measure of the velocity gradient near the wall. However, since $\eta_{99}$ is a function of $K_{1}$, the original form of $\mathrm{K}_{1}$ is more suitable for analyzing real flows.

The friction at the surface will change due to non-equilibrium behavior. The wall friction is given by:

$$
\tau_{\text {wall }}=\mu\left(\frac{\partial v}{\partial x}+\frac{\partial u}{\partial y}\right)=\mu \frac{\partial u}{\partial y}=\frac{\rho^{1 / 2} \mu^{1 / 2} u_{o}^{3 / 2}}{x^{1 / 2}} f^{\prime \prime}(0)
$$

The friction is proportional to the value of $f^{\prime \prime}(0)$ given in figure (1), and the percent reduction in friction due to non-equilibrium behavior is given by

$$
(\% \text { Reduction })=100 \%\left(\left.f^{\prime \prime}(0)\right|_{\mathrm{K}_{1}=0}-f^{\prime \prime}(0)\right)=100 \%\left(.3321-f^{\prime \prime}(0)\right)
$$

The percent reduction in friction for a plate with a chord of 50 microns, and freestream conditions of a $u_{0}$ of $100 \mathrm{~m} / \mathrm{s}$, a pressure of 0.1 atmospheres, and a temperature of $298 \mathrm{~K}$, is show as figure 5 :

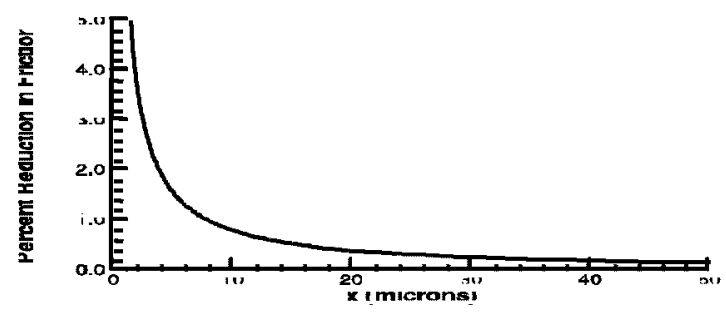

Figure 5. Percent Reduction in Friction

These results show that the reduction in friction will be very large in the initial portion of the plate, and still may be measured downstream on the plate. 
There are two limitations in using this simple model to study flow around a MEMS scale flat plate. The first restriction is the singularity that appears at $\mathrm{x}=0$. Typically this problem is solved by combining the Blasius solution with a Stokes flow solution at the leading edge of the plate. Because the Stokes flow region scales with plate thickness, it becomes less significant in boundary layer growth as the plate thickness decreases to the order of one micron. Figure 6 shows the contours of x-velocity for freestream conditions of a velocity of $100 \mathrm{~m} / \mathrm{s}$, a pressure of 0.1 atmospheres, and a temperature of $298 \mathrm{~K}$.

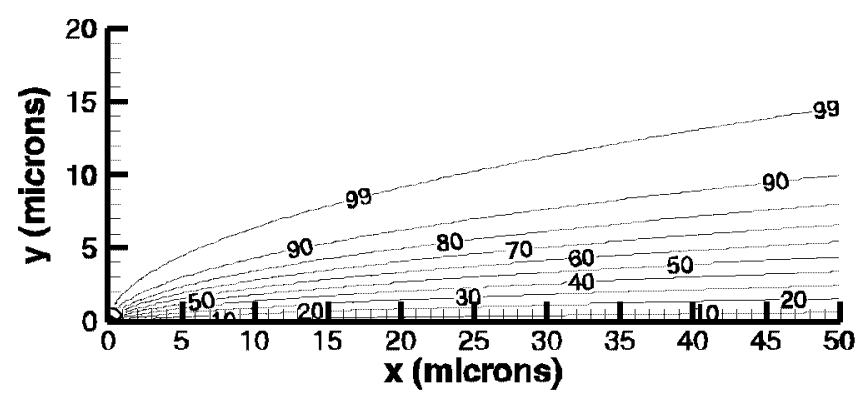

Figure 6. X-Velocity contours for $\mathrm{u}_{\mathrm{o}}=100 \mathrm{~m} / \mathrm{s}, \mathrm{P}=0.1 \mathrm{~atm}, \mathrm{~T}=298 \mathrm{~K}$

The next concern in the Blasius model is the importance of the velocity gradients in the $\mathrm{x}$-direction. Equation (2) can only be used to describe the flow field when $\partial^{2} u / \partial y^{2}$ is much larger than $\partial^{2} u / \partial x^{2}$. Figure 7 shows the ratio of these derivatives, suggesting that the solution is valid for all but the extreme leading edge of the plate.

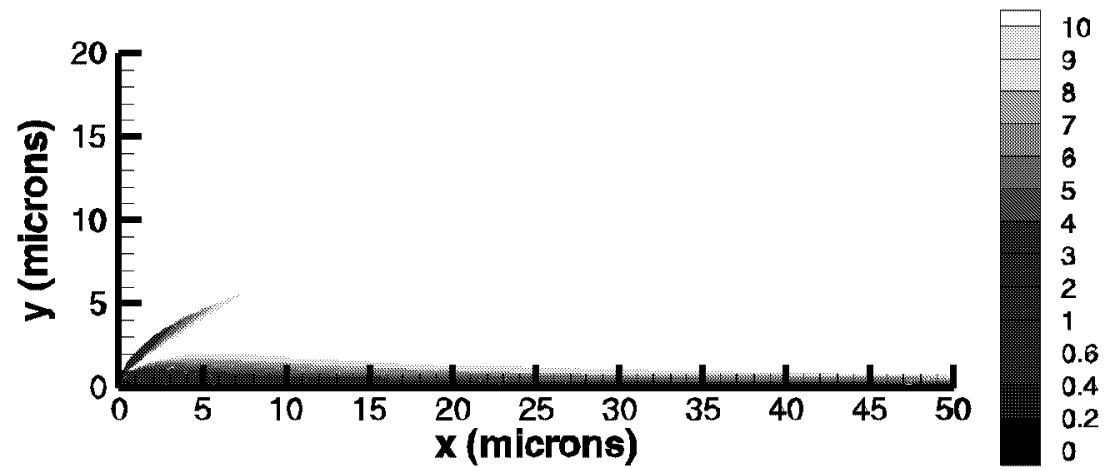

Figure 7. Ratio of $\partial^{2} u / \partial y^{2}$ versus $\partial^{2} u / \partial x^{2}$ for $u_{0}=100 \mathrm{~m} / \mathrm{s}, P=0.1 \mathrm{~atm}, T=298 \mathrm{~K}$

\section{CONCLUSIONS}

The results show that the boundary layer equations can be used to study flow at the MEMS scale, and to judge when non-equilibrium effects become important. While the self-similarity of the Blasius boundary layer is lost, the boundary layer equations continue to provide useful information to study the effects of rarefaction on the shear stress and structure of the flow. They also show the weakness of using a simple geometric Knudsen number in describing the flow, and provide a new flow parameter, $K_{1}$, for describing non-equilibrium behavior.

A Navier-Stokes flow solver [3], incorporating the effects of slip conditions at the boundary, is being used to study the accuracy of these solutions for flow over thin flat plates. 
These results are being used to evaluate test conditions for an experimental study of MEMS scale airfoils. The results of this model, and additional computational studies, suggest that the reduction in drag due to these effects should be measurable for flat plates with chords of $10-40 \mu \mathrm{m}$, at pressures ranging from 0.1 to 1.0 atmospheres.

\section{ACKNOWLEDGMENTS}

The authors gratefully acknowledge support for this work from the Air Force Office of Scientific Research through MURI grant F49620-98-1-0433.

\section{REFERENCES}

1. Panton, Ronald L., Incompressible Fluid Flow, John Wiley and Sons, New York, 1996, pp. 581-591.

2. Gad-el-Hak, Mohamed, Journal of Fluids Engineering 121, 5-33 (1999).

3. Fan, J., Boyd, I.D., Cai, C.P., Hennighausen, K. and Candler, G.V., "Computation of Rarefied gas flows around a NACA 0012 Airfoil," AIAA, 99-3804, 1999. 